REVIEW ARTICLE

\title{
COMPLEMENTARY AND AlTERNATIVE MEDiCine: WHAT IS IT GOOD FOR?
}

\author{
Sunita Dahiya* and D.N. Banerjee \\ Indian Board of Alternative Medicines (IBAM), Kolkata 700020, West Bengal, India \\ *E-mail: drsunitadahiya@gmail.com \\ Tel.: +919630229885.
}

Received: Sep 11, 2016 / Revised: Dec 01, 2016 / Accepted: Dec 02, 2016

\begin{abstract}
Today the plant based medicines are being used globally as home remedies and offering a broad spectrum of activity since ancient times. In the contemporary times, we all know that chemical medications for therapeutic cures have become more and more available in the market. In fact, the pharmaceutical business is regarded as one of the most booming industries in the world, with a high growth capability year on year. Against this, we have also overheard about the herbal remedies used as medications, as suggested by the elders in the family for a variety of common ailments. Present article reports that the huge acceptance and popularity that the modern complementary and alternative medicine (CAM) has gained currently; is originally the rebirth of old health care system and can be explored by expanding the frontiers of the ancient medicine system.
\end{abstract}

Key words: Complementary medicine, Alternative medicine, Ancient health care system, Health benefit.

\section{INTRODUCTION}

The usage of herbs to treat a variety of different ailments is universal, and exists in every human culture on Earth. Despite this, the largest use of medical herbs still occurs in societies which are not fully industrialized. Because of the high costs involved with manufacturing modern medicines, many people living in developing nations simply do not have the financial resources to pay for them, and as a result, they are forced to use natural herbs as an affordable alternative. In recent years, many people living in industrialized countries have begun taking a second look at herbal medicines due to the rising cost of medicine and healthcare in their own nations. There are a number of herbal systems that dominate the world today, and these systems are Chinese herbs, Ayurvedic medicine, Roman and Greek herbs, and Shamanic herbs (Figure 1). Many of the pharmaceuticals which are currently used can be traced back to herbal remedies which were developed many centuries ago. The WHO has indicated that as many as

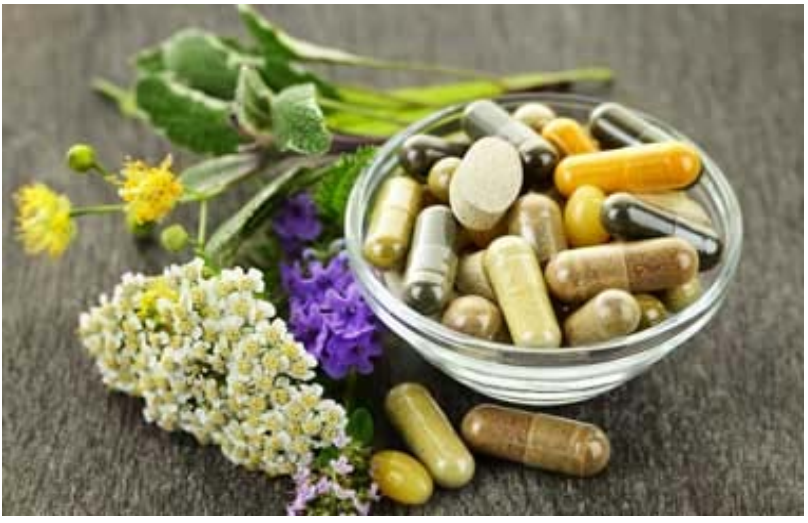

Fig. 1. Herbs and herbal products used as CAM

$80 \%$ of all people living in the world make use of herbal medicine as their main source of healthcare. Because half the world's population (roughly 3 billion people), live on less than $\$ 2$ per day, it is next to impossible for them to buy even basic medicines. Even in the developed nation such as United States, the alternative health industry has exploded within recent years, and an intense global search is being made 
for plants which can be used to create different dietary supplements. Botanists, pharmacologists, and microbiologists are looking in different parts of the globe to find natural chemicals that can be used in the treatment of numerous diseases. Natural remedies can be used to efficiently to treat a range of ailments through correct and regular usage whether it is to boost your immune system, curing many common disease conditions, preventing avoiding hair loss, treating persistent acne or dandruff, treating aches, pains or cuts and burns. These are also very widely and effectively used as medicinal, cosmetic, hygienic and wellness products as potential alternatives to marketed commercial products. The medicament present in many home remedies is in the form of alkaloids, essential oils, enzymes, trace elements and minerals. Once absorbed they are assimilated only in the quantity needed by the human body.

The use of natural products with therapeutic properties is as ancient as human civilization and, for a long time, mineral, plant and animal products were the main sources of drugs (de Pasquale, 1984). The Industrial Revolution and the development of organic chemistry resulted in a preference for synthetic products for pharmacological treatment. The reasons for this were that pure compounds were easily obtained, structural modifications to produce potentially more active and safer drugs could be easily performed and the economic power of the pharmaceutical companies was increasing. Furthermore, throughout the development of human culture, the use of natural products has had magical-religious significance and different points of view regarding the concepts of health and disease existed within each culture. Obviously, this approach was against the new industrialized western societies, in which drugs from natural resources were considered either an option for poorly educated or low income people or simply as religious superstition of no pharmacological value. Among all forms of natural medicines, CAM is gaining continuous importance.

\section{Complementary and alternative medicine Alternative medicine}

It is any practice that is put forward as having the healing effects of medicine, but does not originate from evidence gathered using the scientific method, is not part of biomedicine, or is contradicted by scientific evidence or established science. It consists of a wide variety of health care practices, products and therapies, ranging from being biologically plausible but not well tested, to being directly contradicted by evidence and science, or even harmful or toxic. Examples include new and traditional medicine practices such as homeopathy, naturopathy, chiropractic, energy medicine, various forms of acupuncture, traditional Chinese medicine, Ayurvedic medicine, Sekkotsu, and Christian faith healing. The treatments are those that are not part of the sciencebased healthcare system, and are not clearly backed by scientific evidence.

\section{Complementary medicine}

It is alternative medicine used together with conventional medical treatment, in a belief not confirmed using the scientific method that it "complements" (improves the efficacy of) the treatment. CAM is the abbreviation for complementary and alternative medicine. Integrative medicine (or integrative health) is the combination of the practices and methods of alternative medicine with the conventional medicine.

In general terms, CAM is defined as anything not allopathic. It involves a focus on holistic, individual, and preventative health care whereas alternative medicine is used in place of prescriptions or traditional medicine; complementary medicine is used in addition to other treatments. Treatment methods are considered complementary or alternative because there is insufficient data to prove their safety or effectiveness. This type of care is "based on the patient's needs and must be individualized to match the patient's state, predicament, and preferences with consideration of the best available evidence" (Barnes et al 2008).

Historically, CAM has been in use for thousands of years. From the perspective of modern medicine, CAM has been known as folk medicine, tentative therapy, or the home remedy. However, before the existence of modern medicine, complementary and alternative medicine was the only health care available. It took many forms. It was familial, religious, cultural, regional, and tribal. It was based on belief and experience but little scientific evidence. CAM is described as an assortment of varying health care practices that exist outside of the definition or accepted practices of conventional medicine. Complementary denotes use with conventional medicine. Alternative 
designates practices that occur by themselves, without any additional or complementary conventional medicine. These practices may include therapies, health care systems, and products. One of the example is of the plant based medicines which are being used globally as herbal remedies and offering a broad spectrum of activity since ancient times. In the contemporary times, we all know that chemical medications for therapeutic cures have become more and more available in the market. In fact, the pharmaceutical business is regarded as one of the most booming industries in the world, with a high growth capability year on year.

\section{Why do people use CAM?}

People try CAM for a variety of reasons, including:

- Conventional medicine has not helped solve their medical problem.

- They believe that products derived from nature are healthier and safer than prescription drugs, even though they may not be.

- They like the holistic approach taken by CAM therapists. A holistic approach involves paying attention to all of a client's needs to help her regain and maintain her health. These include not just physical but also emotional, social, and spiritual needs.

Although something can be said for all of these reasons, you should be aware of some of the downsides of using CAM treatments, such as:

- No CAM treatment has been proven to work beyond a shadow of a doubt.

- Some CAM products, although derived from plants, can cause health problems. For instance, ephedra, a Chinese herbal product, was being sold in the United States to help people lose weight and to enhance athletic performance. Because ephedra increased the risk of heart problems and stroke, the U.S. Food and Drug Administration (FDA) banned the sale of ephedra.

- Some CAM products interfere with how prescription drugs work. For instance, St. John's wort, which some people take to treat depression, can interfere with the actions of drugs for treating HIV, cancer, and other diseases. It may also reduce the effectiveness of birth control pills.

- Some herbal products, such as black cohosh, are unsafe to use during pregnancy. The safety of many other herbal products, either during pregnancy or breastfeeding, has not been studied.

- Some people might use an unproven CAM treatment that may not work or may carry risks, instead of a conventional treatment that is known to be effective.

If you choose to try a CAM treatment, be sure to discuss it first with your doctor. Your doctor should know whether the therapy may be helpful and is safe to try along with your current treatments. Some people don't mention their use of CAM treatments to their doctor because they think that their doctor will have negative feelings about CAM. If you are in this situation and would like to try a CAM treatment, perhaps you may want to find a doctor that you feel more comfortable talking to about this.

\section{CAM: Merits and demerits Merits of CAM}

CAM therapies are rooted in deep ancient science

As origins in primeval medicine, also known as Ayurveda; in fact, the Ayurveda is the oldest form of medicine known to mankind, and in modern times has gained new prominence with greater than before research in this space. In fact, the formulations are only recent in nature, and are still in their very primary stages of development when compared with the herbal cures. Preparation of the herbal medicines is a discipline.

\section{Carry little to no side effects}

The commercial therapeutics has exposed mankind to a plethora of possible side effects, including local and systemic effects. When we compare them to herbal formulations, the latter have been proven as safer, and better tolerated amongst all age groups of patients- extending from very young to very old.

\section{No resistance has been reported}

The artificial drug preparations, especially antibiotics, have met with resistance, with the disease causing agents becoming smarter and developing ways and tricks that they stop acting effective anymore. No such problems have been reported with herbal homemade remedies. For example, ginger juice is used very widely for throat infections and throat irritations. Even in individuals who use this daily, reports that the bacteria no longer prone to its effects have not been reported at all. 
Can be administered safely for longer periods of times

Because of the low to nil incidences of side effects, and no reports of resistance, these herbal formulations can be safely administered for longer periods of times. For example, we may not be able to give long term laxatives to those who suffer from chronic constipation as they are bundled with serious side effects, however Psyllium husk when taken in the right quantities, can be administered daily for even months without any real side effects.

\section{Cost effective and can be prepared at home}

It is unquestionably easier to prepare green basil tea at home for a nasty cold, over preparing a combination of drugs which can be really done only in a factory setup. Besides, these preparations also help in the saving the costs.

\section{Demerits of CAM}

Inappropriate for many conditions

Modern medicine treats sudden and serious illnesses and accidents much more effectively than herbal or alternative treatments.

An herbalist would not be able to treat serious trauma, such as a broken leg, nor would he be able to heal an appendicitis or a heart attack as effectively as a conventional doctor using modern diagnostic tests, surgery, and drugs.

\section{Lack of dosage instructions}

Another disadvantage of herbal medicine is the very real risks of doing yourself harm through self-dosing with herbs. While you can argue that the same thing can happen with medications, such as accidentally overdosing on cold remedies, many herbs do not come with instructions or package inserts. There's a very real risk of overdose.

\section{Poison risk associated with wild herbs}

Harvesting herbs in the wild is risky, if not foolhardy, yet some people try to identify and pick wild herbs. They run a very real risk of poisoning themselves if they don't correctly identify the herb, or if they use the wrong part of the plant.

\section{Medication interactions}

Herbal treatments can interact with medications. Nearly all herbs come with some warning, and many, like the herbs used for anxiety such as Valerian and St. John's wort, can interact with prescription medication like antidepressants. It's important to discuss your medications and herbal supplements with your doctor to avoid dangerous interactions.

\section{Lack of regulation}

Because CAM therapies are not tightly regulated, consumers also run the risk of undergoing improper treatment or buying inferior quality herbs. The quality of herbal products may vary among batches, brands or manufacturers. This can make it much more difficult to prescribe the proper dose of the herb.

However, CAM represents an important part of healthcare delivery and provision in global scenario. It is a rapidly growing part of the economy, used by significant numbers of citizens in the different parts of the world.

CAM should be included in the strategic approaches to health promotion and prevention but it is currently largely ignored by those who make health policy. CAM can significantly contribute to answer the needs of community as improving public health, enabling healthy ageing, providing affordable health systems, reducing antimicrobial resistance and redressing health inequalities.

\section{CAM treatments found in nature}

Some CAM treatments use substances found in nature, such as herbs, vitamins, and minerals. The idea that natural substances might be used as medicines is not new. Practically since the beginning of time, people have used parts of plants and animals to treat diseases.

In fact, some conventional drugs come from nature. For instance, aspirin is derived from a substance found in the bark of the willow tree. Some CAM products are sold as dietary supplements. These are products taken by mouth that are intended to supplement, or add to, the diet. They come in many forms, including tablets, teas, and powders. Table 1 enlists some of the widely used practitioner based or nonpractitioner based CAM therapies.

\section{Key characteristics of CAM}

A number of key elements are characteristic of the CAM approach to healing. They include:

\section{Holistic approach}

Most Complementary and Alternative Medicine practices are based on a holistic, or 'whole person' approach, i.e. how the physical, mental, emotional, and spiritual elements of an individual are interconnected to maintain or 
regain wellness and health.

Holistic approaches focus on the whole individual person rather than just on the illness or a diseased part of the body. They fully involve the patient in the diagnosis and management of his/her illness. Many aspects of a patient's life may influence a health problem and understanding the 'whole individual environment' helps to develop a successful treatment plan. The aim of holistic therapy is to restore harmony of body, mind and spirit.

Table 1. Some popular CAM therapies

\begin{tabular}{|c|c|}
\hline Practitioner based CAM & Non-practitioner based CAM \\
\hline Acupuncture* & Deep breathing exercises \\
\hline Ayurveda* & Diet-based therapies \\
\hline Biofeedback $^{*}$ & Guided imagery \\
\hline Chelation therapy* & Homeopathic treatment \\
\hline Chiropractic or osteopathic manipulation* & Meditation \\
\hline Energy healing therapy/Reiki* & Movement therapies \\
\hline Hypnosis* & Natural products \\
\hline Massage* & Progressive relaxation \\
\hline Naturopathy* & Qi gong \\
\hline Traditional healers* & Tai chi \\
\hline & Yoga \\
\hline & and other product \\
\hline
\end{tabular}

\section{Health as a dynamic rather than static state}

Homeostasis, as currently defined, is a selfregulating process by which biological systems maintain stability while adjusting to changing conditions. This concept explains how an organism can maintain more or less constant internal conditions that allow it to survive in the face of a changing and often hostile external environment. When confronted with physiological and mental/emotional stress, a healthy organism is able to mount a protective response, to reduce the potential for harm, and restore an (adapted) equilibrium. If this coping strategy is not successful, illness may result.

This perspective on health and disease is a central tenet of the CAM approach and has lately gained acclaim in a proposal in a mainstream medical journal for a new definition of health as "The ability to adapt and self-manage in the face of social, physical, and emotional challenges' (Huber et al 2011). Rather than health being defined solely as the absence of disease, this dynamic definition focuses on resilience or the capacity to cope which maintains and restores a person's integrity, equilibrium, and sense of wellbeing.

\section{Assisting the person's innate healing capacity}

Human beings are considered as whole, adaptable living systems whose innate constitutional vitality and resistance to disease can be stimulated, supported and strengthened to maintain or regain health. CAM therapies are mainly directed towards reinforcing the resilience, resistance and immune status of the individual concerned thereby reducing the susceptibility to illness and disease as well as addressing any already existing disease process. As such CAM approaches are not limited to simply addressing certain diseases but are universally applicable to patients suffering from all kinds of diseases. Such treatment can be used complementary to conventional medical intervention that is more disease focused.

\section{Individualised healthcare and treatment}

CAM therapies vary to suit individual needs instead of being used to treat specific diseases regardless of the individual who is suffering; the focus is on treating the person rather than the condition. Taking account of the patient's constitutional nature and social context as well as the individual response to any affliction enables the doctor/practitioner to adjust and individualise the treatment strategy throughout a course of treatment for optimum effect. A more individualised approach has lately also been acknowledged in mainstream medicine and has become known as 'personalised medicine', which however more narrowly refers to molecular biologic specifications in individuals rather than to a response to individual patient needs as is understood in the concept of person-centred medicine. 


\section{Salutogenesis}

Salutogenesis is a term coined by Aaron Antonovsky, a professor of medical sociology. The term describes an approach focusing on factors that support human health and wellbeing, rather than on factors that cause disease. More specifically, the 'salutogenic model' is concerned with the relationship between health, stress, and coping (Lindstrom and Eriksson, 2005). Salutogenesis explores the reasons why some people stay healthy in the face of hazardous influences whilst others, faced with similar pathogenic factors or other difficulties, fall ill. Thus the ultimate objective of health promotion is to highlight and facilitate the essential prerequisites for maintaining health. Whilst this approach is only applied in a limited fashion, if at all, within biomedical Practice (Alonso, 2004), it is central to the CAM perspective.

\section{Therapeutic relationship}

A positive functioning partnership between the patient and the healthcare professional engages the patient's innate healing capacity and provides motivation to make healthy lifestyle changes. Such a positive therapeutic relationship should not be dismissed as a 'placebo effect' or a 'good bedside manner'. In their encounter with CAM providers, citizens particularly value the following:

Empathetic communication in consultations with more time available than in biomedical encounters.

Involvement in their own care through participation in decision-making about their treatment options and the provision of selfhelp strategies.

Whole person approach and person-centred healthcare.

Explanatory frameworks within which to explore health and illness, which are frequently congruent with citizens' own ideas about health and illness. Patients report a high satisfaction rating from this kind of encounter (Nissen et al 2012).

\section{Prevention, self-care, health literacy and patient empowerment}

Staying healthy and preventing disease requires the development of personal responsibility and involvement. The concept of self-care requires a conscious focus on and understanding of one's physical, mental and emotional state and the ability to take corrective action when necessary. Helping patients to develop sufficient levels of self-awareness and the know-how required to change unhealthy patterns of behaviour to improve their health is remarkably empowering for the patient. In the first instance, this will enable a person to self-correct a relatively minor health problem. If the condition is more serious it may be necessary to consult a healthcare professional who can work with the patient to take the steps required to recovery. In this way, the patient is not a passive participant; the patient and healthcare professional cooperate as partners.

\section{Short descriptions of individual cammodalities (Christy, 2014) Acupuncture}

Acupuncture is perhaps the best-known aspect of traditional Chinese Medicine (TCM) in the west. It aims to influence body functions and stimulate and restore the body's own regulatory system by using specific (acupuncture) points on the surface of the body. Besides the use of needles, the application of pressure (acupressure) and heat (moxibustion) are also traditionally used. Diagnosis and treatment are conducted in accordance with the presenting individual pattern of disharmony and are based on traditional concepts, centuries of clinical experience and on modern scientific basic research. Acupuncture can treat a wide range of complaints both organic and functional in origin. It is also well known for its application in pain management and modern research has confirmed its effectiveness in the treatment of low back pain, depression, the treatment of migraine and many other common conditions (NICE, 2009; Hopton et al 2014; Collins, 2012). Acupuncture can be combined with other therapeutic TCM techniques such as moxibustion, cupping, guasha (a kind of rubbing therapy), herbs, dietetics, tuina (massage and manual therapy) and qigong (exercises, breathing, concentration). Acupuncture is often integrated in various therapeutic aspects of mainstream medicine.

\section{Ayurveda}

Ayurveda (the 'science of life') is a system of traditional medicine native to the Indian subcontinent using methods for achieving physical, mental and spiritual health and wellbeing. Ayurveda emphasises prevention and a holistic approach to therapy and is practised as a 
form of CAM within the western world, where several of its methods, such as the use of herbs, massage, and yoga are applied on their own as a form of CAM treatment.

\section{Anthroposophic medicine}

Anthroposophic medicine is a holistic and salutogenetic approach to medicine focusing on strengthening the patient's organism and individuality. The self determination, autonomy and dignity of patients are central themes. Therapies are intended to enhance a patient's capacities to heal and include anthroposophic medicines as well as various art therapies like painting and sculpture therapy, music, singing and speech therapy, physiotherapy and massage, psychotherapy, curative education and social therapy - and eurythmy therapy in which special body movements are employed for therapeutic purposes.

\section{Chiropractic}

Chiropractic is a healthcare profession that focuses on disorders of the musculoskeletal system and the nervous system, and the effects of these disorders on general health. Chiropractic care is used most often to treat neuromusculo skeletal complaints, including but not limited to back pain, neck pain, pain in the jointsof the arms or legs, and headaches.

Chiropractic physicians or chiropractors practise a hands-on approach to healthcare that includes patient examination, diagnosis and treatment. They have broad diagnostic skills and are also trained to recommend therapeutic and rehabilitative exercises, as well as to provide nutritional, dietary and lifestyle counselling.

\section{Herbal medicine}

Herbal medicine - also called phytomedicine or phytotherapy - refers to using a plant's seeds, berries, roots, leaves, bark, or flowers for medicinal purposes. Whole herbs contain many chemical constituents working synergistically together to treat disease and support the body's own healing mechanisms (e.g. its immunity). Herbal medicine practice is rooted in hundreds of years of experience of using plant medicines which today is underpinned by the scientific study (pharmacognosy) of plant medicines and their chemical constituents. Herbal medicine is becoming main stream as improvements in analysis and quality control along with advances in clinical research show the value of plant medicines in the treatment and prevention of disease Apart from the herbal medicine itself, herbal practitioners will routinely offer advice on appropriate changes of lifestyle, diet and the adoption of stress-reduction techniques and exercise.

\section{Homeopathic medicine}

Homeopathy is a whole medical system that originated in Germany. The fundamental idea of homeopathy is the Similarity Principle, which implies that substances capable of causing disorder in healthy subjects are used as medicines to treat similar patterns of disorder experienced by ill people. Homeopathic medicines are aimed to direct and stimulate the body's self-regulatory mechanisms. Homeopathy is highly individualized while taking into account the symptoms and signs of the disease, the patient's physical build, personality, temperament and genetic predispositions. Apart from homeopathic medication, advice on change of lifestyle, diet and substance-abuse behaviours, acquisition of stress-reduction techniques and exercise are part of the package of care.

\section{Kinesiology}

Kinesiology is a uniquely client-specific approach to health and wellbeing. It uses 'Muscle Response Testing' to get feedback from the body at a sub-conscious, reflexive level. In response to gentle pressure, a locked muscle is seen as a positive response to an 'input stress' such as a statement, a memory, a food or an educational activity. A weak or unlocked muscle indicates a negative response, a lack of ease. Applied Kinesiology was first developed as a therapy in the 1960's by Dr. George Goodheart supported in his research by around 18 other chiropractors. One of these, Dr. John Thie offered the Touch for Health Kinesiology Synthesis, a sub-set of Applied Kinesiology to the general public. From there, kinesiology developed as a broad based stand-alone therapy.

During a kinesiology session the mental, chemical, physical, energetic and environmental aspects of a problem are dealt with simultaneously. Kinesiology offers a selection of techniques and remedies drawn from chiropractic, traditional Chinese medicine and other sources to support healing.

\section{Naturopathic or traditional european medicine (TEM\&N)}

Rooted in Hippocratic, Mediterranean and middle-European traditional healing systems, 
TEM\&N currently represents a pragmatic blend of traditional, scientific and empirical methods in the prevention and treatment of illness and the maintenance of health. The principles and methods of TEM\&N are taught so they can be adopted and practised by those seeking better health or health maintenance. The principles involved include:

Understanding the human being as an integral part of nature achieving optimum health by having body, mind and spirit in harmony with the environment.

Understanding and employing the inherent homeostatic and regulatory principles of the body's biological and biochemical and electromagnetic functions.

Understanding the central importance of the interplay of the elements, temperament and constitution so that treatment is suited to individual need.

Making an individual diagnosis based on the unique analysis of each case.

Highlighting the necessary measures to achieve full health and prevention of disease. This includes appropriate individualised dietary and lifestyle advice suited to the specific individual.

TEM\&N is a distinct healthcare profession, emphasizing the importance of prevention as well as offering treatment through the use of a variety of therapeutic methods and natural substances that encourage individuals' inherent self-healing eliminative and constructive processes to obtain optimum health. TEM\&N professionals comprehend human beings and other living creatures as a vital individual unity of body, soul and spirit.

Current methods of practice include: cupping, massage, treatment with leeches, bloodletting, spagyric therapy, apitherapy, balneotherapy, hydrotherapy, relaxation exercises, movement therapy, reflexology, neural therapy, nutritional advice, vitamin and mineral therapy, fasting, herbalism/phytotherapy, the use of essential oils, psychological counselling, stress management, lifestyle regulation and others.

\section{Osteopathy}

Osteopathy is a system of medicine that emphasizes the theory that the body can heal itself given normal, healthy structural relationships, environmental conditions, and nutrition. Treatment attends to body mechanics and manipulative methods in diagnosis and therapy. It is a contact and patient-centred healthcare discipline, that emphasises the interrelationship of structure and function of the body, facilitates the body's innate ability to heal itself and supports a whole-person approach to all aspects of health and healthy development, principally through manual treatment. The practice of osteopathy uses current medical and scientific knowledge to apply the principles of osteopathy to patient care. Scientific plausibility and evidence-based outcomes have a high priority in patient treatment and case management.

Osteopathy provides a broad range of approaches to the maintenance of health and the management of disease. It embraces the concept of the unity of the individual's structure (anatomy) and function (physiology); as such osteopathy is a patient-centred rather than disease-centred system of healthcare. An essential component of osteopathy is its great attention to body mechanics and its manual methods in diagnosis and therapy. Osteopathy was developed as a means to facilitate normal self-regulating/self-healing mechanisms in the body by addressing areas of tissue strain, stress or dysfunction which may impede normal neural, vascular and biochemical mechanisms.

\section{Reflexology}

Reflexology is the study and practice of treating points and areas in the feet and hands that relate to corresponding parts of the body. Using precise hand and finger techniques, a reflexologist may improve circulation, induce relaxation and enable homeostasis. This encourages the person's own healing systems to be activated to maintain well being.

Reflexology has been practiced for over 4000 years, its practice dates back to Ancient Egypt, India and China. It was introduced to the West by a surgeon, Dr. William Fitzgerald, who published an article in 1917 on Zone Therapy or Relieving Pain at Home. In the 1930's a physiotherapist, Eunice Ingham, further developed his theory which become known as reflexology.

Reflexology is a holistic treatment tailored to the individual that can be received by anyone at any age, from newborn babies to the elderly, although there may be times when it is not a suitable treatment. Reflexology is often used alongside allopathic healthcare to increase health and wellbeing. Professional reflexologists do not claim to cure, diagnose or prescribe. 


\section{Shiatsu}

Shiatsu is an autonomous natural healthcare system which originated in Japan, and is influenced by traditional Chinese medicine and more recently Western knowledge. It derives its theoretical and practical roots from the ancient traditions of East Asian philosophies and healing and the holistic understanding of life and health based upon them.

Its aim is to stimulate and support the processes of natural self-healing, well-being and personal growth, and to maintain health, through balancing the energetic system of a person. The method uses the application of pressure or energetic touch on specific points, channels (meridians) or areas of the body to balance the circulation of the body's energy (Ki in Japanese or Qi in Chinese).

Treatment involves brief or sustained pressure with thumbs, hands, elbows, knees and feet to meridians and tsubo (pressure points) and to other physical structures. Treatment may also include rotations and stretching of limbs, joints and meridians. Shiatsu is usually given on a soft mat on the floor. Shiatsu enhances selfawareness and sensitivity, and releases tension. It also supports and nourishes a person's lifeforce. Applied by a practitioner with appropriate training, shiatsu promotes general well being and can be used as a preventative healthcare method. It can also assist people through crisis, difficult life phases and processes of change. It can treat people presenting with a widevariety of physical conditions.

\section{Traditional Chinese medicine (TCM)}

Traditional Chinese medicine (TCM) originated in ancient China and has evolved over thousands of years. It encompasses many different practices, including acupuncture, moxibustion (burning an herb above the skin to apply heat to acupuncture points), Chinese herbal medicine, tuina (Chinese therapeutic massage), guasha (a rubbing therapy), dietary therapy, and tai chi and qigong (practices that combine specific movements or postures, coordinated breathing, and mental focus).

Traditional Chinese medicine dates back more than 2,500 years. Traditional systems of medicine also exist in other East and South Asian countries, including Japan (where the traditional herbal medicine is called Kampo) and Korea. Some of these systems have been influenced by TCM and are similar to it, but each has developed distinctive features of its own.

\section{Tibetan medicine}

Tibetan medicine, Sowa Rigpa (the 'science of healing'), is a system of traditional medicine native to Tibet (and adapted in parts of India, Nepal, Bhutan, Mongolia, Himalayan regions, Siberia etc.). It is a complete traditional medical system with all divisions of physiology, pathogenesis, general and special pathology, diagnosis and treatment. Based on a threefold humoral theory, body mind concept and Buddhist psychology, Tibetan Medicine is a holistic medicine, which aims to balance the three aspects of life: body, energy and mind by internal and external therapies as well as by the introduction of health promoting selfadministered exercises. It employs a complex approach to diagnosis and utilizes lifestyle and dietary modification, medicines composed of natural materials (e.g. herbs and minerals) and physical therapies (e.g. Tibetan moxibustion \& Tibetan massage therapy etc.) to treat illness.

\section{Yoga}

Yoga is a systematic practice of physical exercise, breath control, relaxation, diet control, positive thinking and meditation aimed at developing harmony in the body, mind, and environment. The practice entails low-impact physical activity, postures (called asanas), breathing techniques (pranayama), relaxation, and meditation. Yoga is an ancient system of physical and mental practices that originated during the Indus Valley civilization in South Asia. The fundamental purpose of yoga is to foster harmony in the body, mind, and environment. The origin of the word 'Yoga'is a Sanskrit word Yog meaning 'union'. Yoga is a union of the organ systems in the body with the consciousness in the mind. Yoga is a low-cost self-help approach to well-being. Regular practice of yoga can lead to reduced stress levels, improved flexibility and muscle strength, improved posture, improved awareness of the physical body and the self. As it is not necessary to be in peak physical condition to practice yoga, it is an ideal activity for sedentary people and for seniors as well as for those who are more active.

\section{CONCLUSION}

Natural remedies can be used to efficiently to treat a range of ailments through correct and regular usage whether it is to boost your immune system, curing many common disease conditions, preventing avoiding hair loss, treating persistent acne or dandruff, treating 
aches, pains or cuts and burns. These are also very widely and effectively used as medicinal, cosmetic, hygienic and wellness products as potential alternatives to marketed commercial products. The medicament present in many home remedies is in the form of alkaloids, essential oils, enzymes, trace elements and minerals. Once absorbed they are assimilated only in the quantity needed by the human body.

\section{REFERENCES}

Alonso Y. The biopsychosocial model in medical research: the evolution of the health concept over the last two decades. Patient Educ. Couns. 2004;53(2):239-44. [DOI: 10.1016/S0738-3991(03)00146-0]

Barnes PM, Bloom B, Nahin RL. Complementary and alternative medicine use among adults and children: United States, 2007. Natl. Health Stat. Report 2008; 10(12):1-23.

Collins S. Acupuncture may be effective for migraines: Experts split on whether to recommend the ancient therapy, 2012.

Christy. CAM 2020: The contribution of Complementary and Alternative Medicine to sustainable healthcare in Europe, 2014.

de Pasquale A. Pharmacognosy: the oldest modern science. J. Ethnopharmacol. 1984;11(1):1-16. [DOI: 10.1016/0378 -8741(84)90092-8]

Hopton A, Macpherson H, Keding A, Morley S. Acupuncture, counselling or usual care for depression and comorbid
Keeping in view extensive potential and increasing popularity associated with usage of CAM therapies, both CAM practices and CAM research seem to have challenging future. Although CAM has attained huge acceptance and popularity; it is originally the rebirth of ancient health care system and can be further explored by expanding the frontiers of the ancient medicine system.

pain: secondary analysis of a randomised controlled trial. BMJ Open 2014;4(5):e004964. [DOI: 10.1136/bmjopen-2 014-004964]

Huber M, Knottnerus JA, Green L, van der Horst H, Jadad AR, Kromhout D, Leonard B, Lorig K, Loureiro MI, van der Meer JW, Schnabel P, Smith R, van Weel C, Smid H. How should we define health? British Med. J. 2011;343:d4163 [DOI: 10.1136/bmj.d4163]

Lindstrom B and Eriksson M. Salutogenesis. J. Epidemiol. Community Health 2005;59(6):440-2. [DOI: 10.1136/jec h.2005.034777]

NICE, Low back pain in adults: early management, Clinical guideline [CG88], 2009.

Nissen N, Johannessen H, Schunder-Tatzber S, Lazarus A, Weidenhammer W. Citizens' needs and attitudes towards CAM. Final Report of CAMbrella Work Package 3, 2012, 1104.

http://www.improvehealthcare.org/complementary-andalternative-medicines 